\title{
Reduction glossectomy of congenital macroglossia due to lymphangioma
}

\author{
Jun Hyeok Kim, \\ Hyo Jeong Kwon, \\ Jong Won Rhie \\ Department of Plastic and \\ Reconstructive Surgery, College of \\ Medicine, The Catholic University of \\ Korea, Seoul, Korea
}

\begin{abstract}
Macroglossia is a rare clinical condition defined as an enlarged tongue. Macroglossia can cause structural deformities like diastema and disproportionate mandibular growth and present functional disorders such as dysarthria, dysphonia, and respiratory problems. A 7-year-old boy who had lymphangiomatous macroglossia was treated with a reduction glossectomy by anchor-shaped combination of a U-shape and modified key-hole resection. Postoperatively, the reduced tongue was contained completely within the oral cavity, but open bite remained due to prognathism. Sensory and motor nerves to the tongue appeared to be intact, and circulation was adequate. This patient will be monitored for recurrence of tongue enlargement.
\end{abstract}

Keywords: Glossectomy / Lymphangioma / Macroglossia

\section{INTRODUCTION}

Macroglossia is an uncommon anatomic deformity [1] defined as pathological enlargement of the tongue [2]. Macroglossia can lead to dysarthria, dysphagia, and obstructive sleep apnea because the movement of the tongue has critical roles in speech, swallowing, and respiration [3]. In addition, an enlarged tongue can cause dento-musculoskeletal abnormalities such as diastema, open bite, and mandibular protrusion.

Macroglossia originates from various causes including idiopathic muscular hypertrophy (Beckwith-Wiedemann syndrome), vascular malformations (angiomas and lymphangiomas), tumors, and endocrine disorders [4]. Among those, lymphangiomatous macroglossia is extremely rare and presents with especially diffuse involvement [5]. This form of macroglossia has a chronic course of progression and reaches a developmental plateau at puberty without spontaneous regression.

Correspondence: Jong Won Rhie

Department of Plastic and Reconstructive Surgery, Seoul St. Mary's Hospital, College of Medicine, The Catholic University of Korea, 222 Banpo-daero, Seochogu, Seoul 06591, Korea

E-mail: rhie@catholic.ac.kr

Received May 10, 2019 / Revised July 24, 2019 / Accepted August 16, 2019
We present a rare case of congenital macroglossia with lymphangioma that presented with disproportionate mandibular growth and dento-alveolar protrusion that was successfully treated with reduction glossectomy.

\section{CASE REPORT}

A 7-year-old boy was referred to our hospital with a chief complaint of tongue enlargement. The degree of symptom was accompanied by his growth. The enlarged tongue was dry and prevented mastication. An enlarged mass in the right submandibular area at birth caused breathing and breastfeeding difficulties. When he reached 3 years of age, he underwent a surgical operation to remove the mass, which was confirmed as lymphangioma by histopathologic examination. At 5 years old, he underwent sclerotherapy with three injections of 95\% alcohol; however, this therapy had no effect on tongue reduction.

On physical examination, the patient had a large globular tongue, $11 \times 8 \times 3 \mathrm{~cm}$ in size (Fig. 1). On palpation, the tongue was firm, pebbly, and non-tender with crenation on both sides. The movement of the tongue was almost totally restricted by its texture and stiffness. The patient had no obstructive sleep ap- 


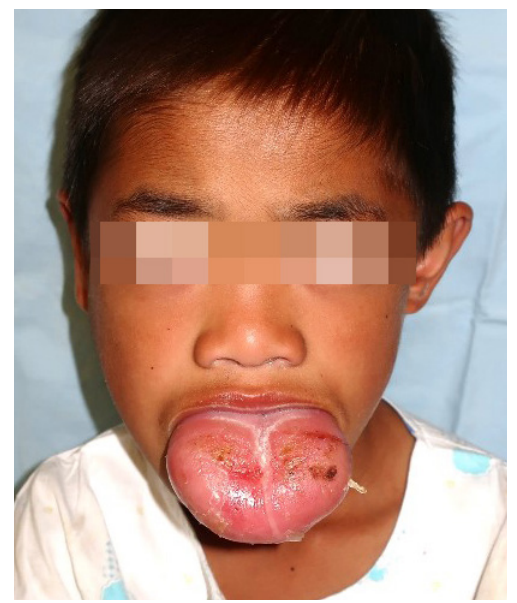

Fig. 1. Clinical photograph before operation.
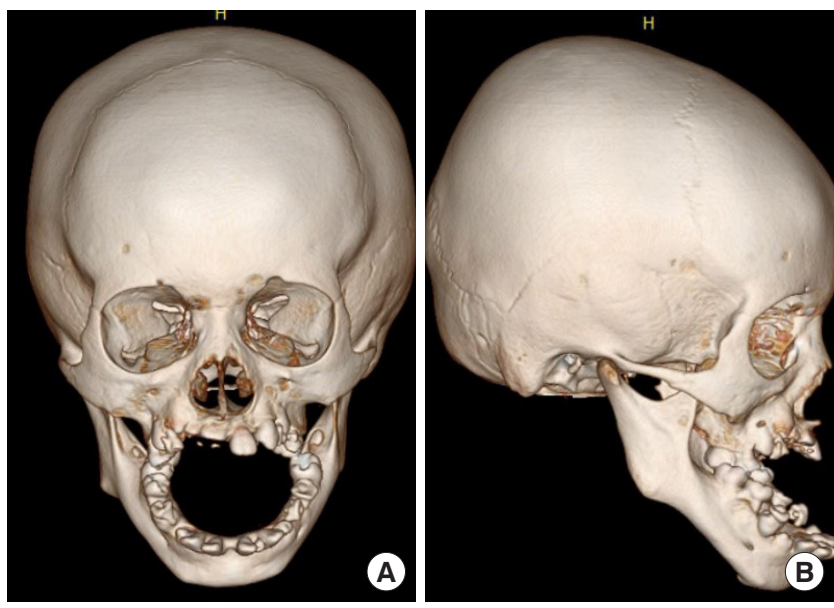

Fig. 2. Preoperative computed tomography revealed prognathism and class III malocclusion. (A) Anteroposterior view and (B) lateral view.

nea but was uncomfortable with oral intake and had limited communication due to dysarthria. The patient was not able to close his mouth properly and was constantly drooling. In preoperative facial bone three-dimensional computed tomography (3D-CT), diastema, tooth spacing, prognathism, and class III malocclusion were noted, but other skeletal deformities were not significant (Fig. 2). Neck CT revealed probable hemangiolymphangioma involvement in both the right parotid gland and right parapharyngeal space.

Reduction glossectomy was planned with anchor-shaped combination of a U-shape and modified key-hole resection (Figs. 3, 4). The incision was executed using a surgical scalpel; hemostasis was completed with electrocautery. The excess tongue muscle tissue was resected. The tongue was closed using absorbable 5-0 Vicryl sutures (Ethicon, Bridgewater, NJ, USA) (Fig. 5). Postoperatively, the reduced tongue was contained completely within the oral cavity, but an open bite remained as

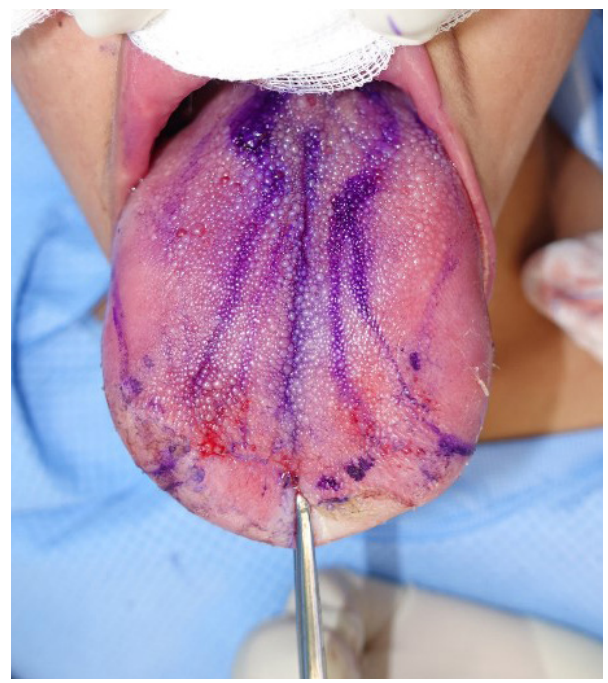

Fig. 3. Design of partial glossectomy by anchor-shaped combination of a U-shape and modified key-hole resection.

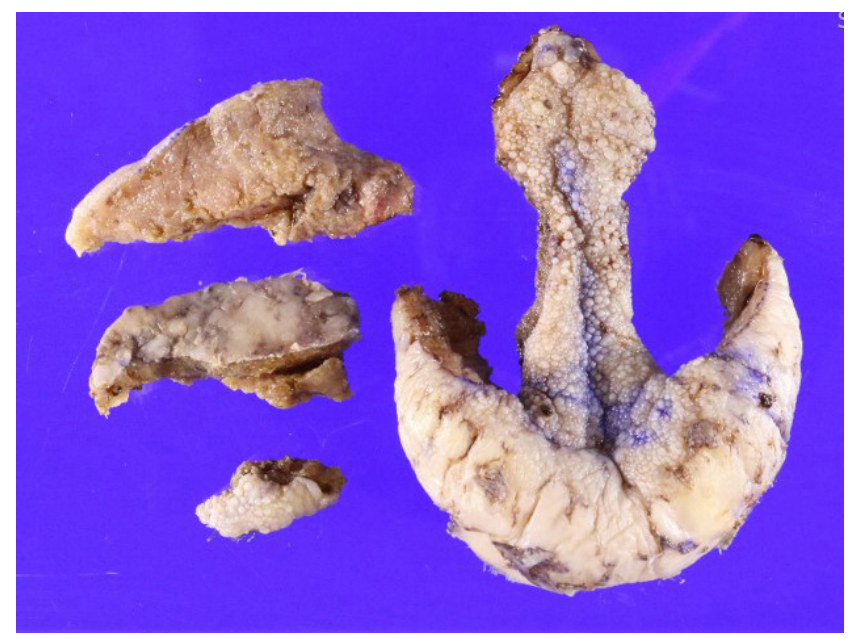

Fig. 4. Gross picture of the resected tissue.

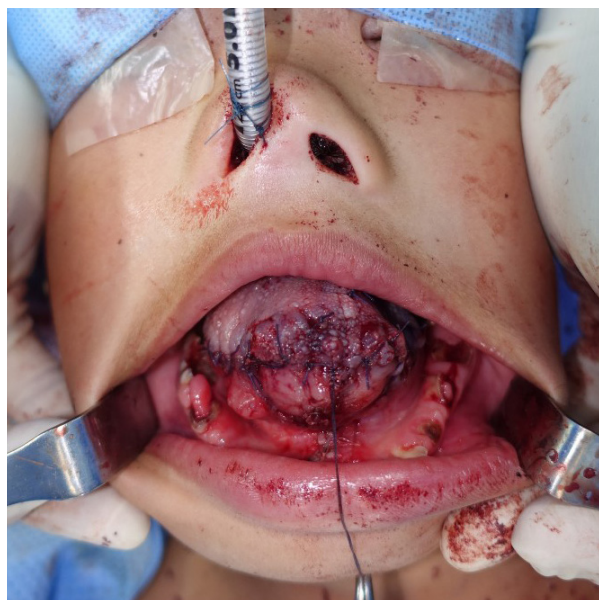

Fig. 5. Immediate postoperative clinical picture: the tongue was contained completely within the oral cavity. In this picture, the tongue was retracted by suture material for visualization. 
the result of increased gonial angle and mandibular plane and disproportionate mandibular growth. Sensory and motor nerves to the tongue appeared to be intact, and circulation was adequate. Based on histopathological analysis, the patient was diagnosed with lymphangioma, consistent with macroglossia (Fig. 6).

\section{DISCUSSION}

Congenital macroglossia is caused by muscular hypertrophy, adenoid hyperplasia, hemangiomas, lymphangiomas, Down syndrome, Beckwith-Wiedemann syndrome, or other causes. Acquired macroglossia is caused by metabolic disorders, such

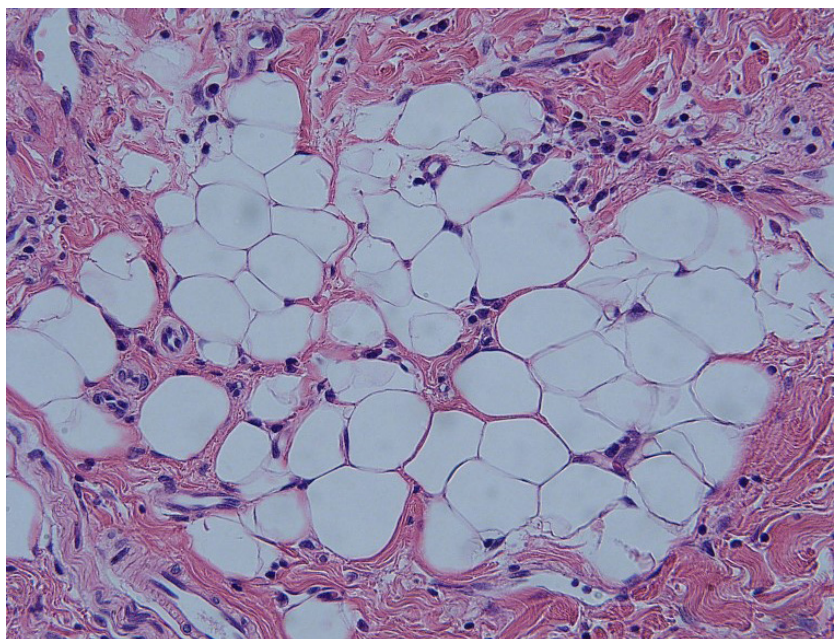

Fig. 6. Pathological examination. Enlarged lymphatic channels in loose connective tissue, disorganized smooth muscle in wall of channels, and peripheral lymphoid aggregates indicate lymphangioma $(\mathrm{H} \& \mathrm{E}, \times 60)$. as hypothyroidism, diabetes mellitus, acromegaly, inflammation, allergic reactions, radiation, and amyloidosis [2].

Lymphangiomatous macroglossia is, inter alia, extremely rare [5]. Lymphangioma is a lymphatic malformation leading to a structural anomaly involving lymphatic channels [6]. Lymphangiomatous macroglossia develops chronically and attains a static stage around puberty; spontaneous regression does not occur. Although the lymphangioma is diffuse and includes the laryngopharynx, respiratory problems seldom occur. Accompanied clinical symptoms are diastema, mandibular protrusion, crenation of the tongue, dysphonia, respiratory problems, and drooling [7]. Radiographic features include increased gonial angle and mandibular plane, disproportionate mandibular growth, and dento-alveolar protrusion [8].

The treatment of macroglossia depends on its etiology and involves treatment of the systemic disease. The most common treatment of macroglossia is glossectomy, and indications for glossectomy include substantial tongue mass, crenated tongue, tongue stretched to the chin or nose tip, dysphonia, and sociopsychological problems due to its effects [7].

Glossectomy techniques can be classified in two groups: peripheral glossectomy and glossectomy along the median line [4]. Both techniques require excision of a part of the mass followed by suturing of the margins. Depending on the type of excision, different sub-types are wedge glossectomy, key-hole glossectomy, or w-shaped glossectomy [2].

Knowledge of lingual neurovascular territories is important for reduction glossectomy. Designs that include the anatomical knowledge avoid injuring either side of neurovascular pedicles. In this case, reduction glossectomy was tried newly with anchor-shaped combination of a U-shape and modified key-hole

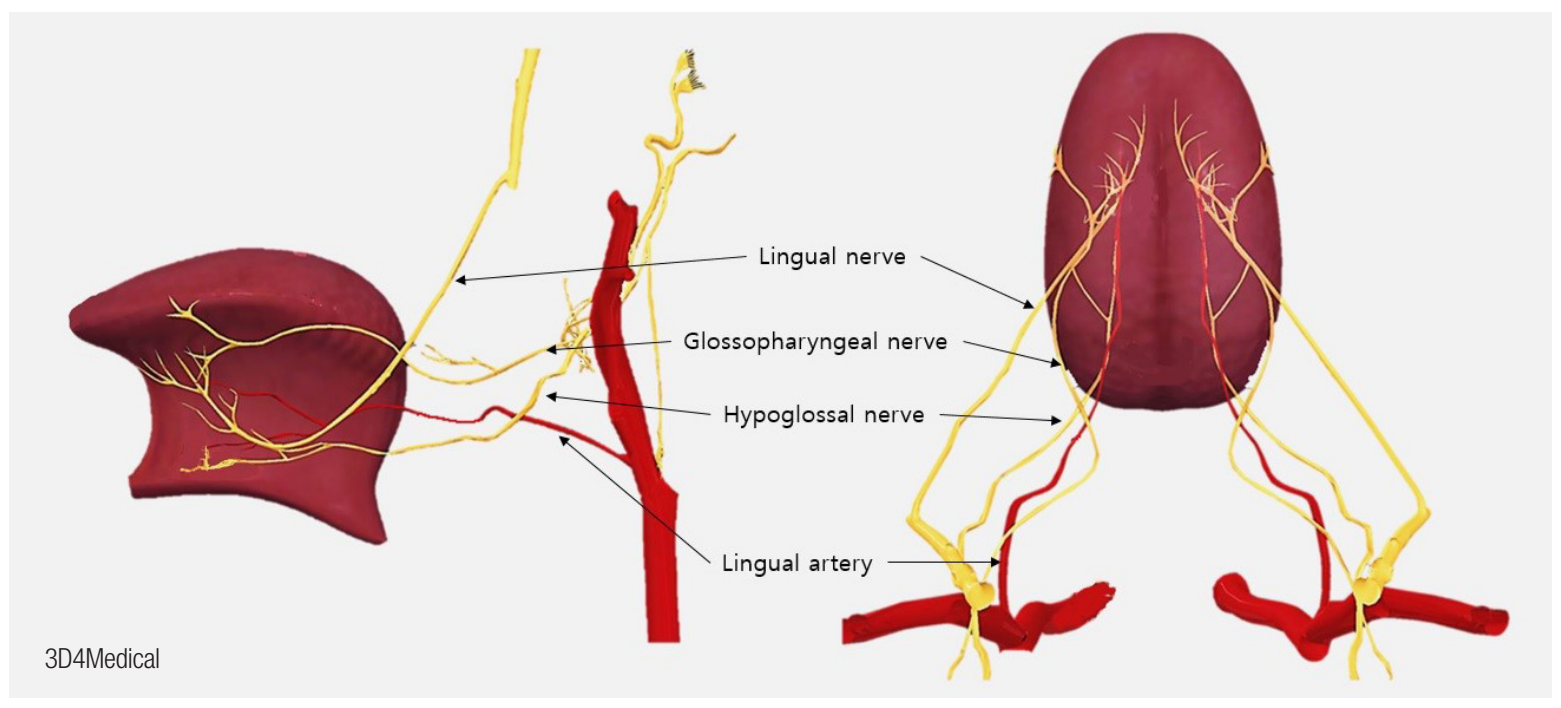

Fig. 7. Schema of the glossal neurovascular anatomy. 
resection. It is also a combination reduction of the periphery and the median line, which effectively enables extensive debulking. U-shaped incision reduced the bulky lateral side, and a modified key-hole resection debulked the central part and shortened the tongue and saved bilateral neurovascular pedicles. And the lower flap of the tongue was preserved because of concerning of injury during debulking of the central and anterior regions.

Lingual vascular territories are dependent on segments of the lingual artery: segment original (segment I), segment within the hyoglossus muscle (segment II) of the dorsal artery, ascending segment (segment III), and horizontal segment (segment IV) of the deep lingual artery [9]. The base and posterior half of the tongue lie on segment III and are nourished by the sublingual and deep lingual arteries. The anterior half lies on segment IV and is supplied by the transverse running of the deep lingual artery. Each artery is responsible for supplying its unilateral territory (Fig. 7).

The sensory innervation of the tongue is supplied by cranial nerves (CNs) V, VII, IX, and X. The special visceral afferent (SVA) of $\mathrm{CN}$ VII and the lingual nerve $\left(\mathrm{CN} \mathrm{V}_{3}\right)$ innervate the taste buds on the fungiform papillae of the anterior tongue. The lingual branch of CN IX supplies the SVA and general sensory afferent (GSA) to the posterior one-third of the tongue, including the circumvallate papillae (Fig. 7); CN X provides both SVA and GSA to the tongue anterior to the epiglottis [10].

Motor innervation of the tongue is provided by $\mathrm{CN}$ XII. The tongue neither has nor is in contact with any bones; only a contracting muscle causes $3 \mathrm{D}$ changes in tongue shape. This ability to shape the tongue provides speech articulation. The single muscle consists of separate neuromuscular compartments, or bellies, having separate terminal nerves. Individual bellies can have different activity than the others of the same muscle. $\mathrm{CN}$ $\mathrm{XII}$ enters the tongue on each side at the ventrolateral aspect of the posterior tongue. After this CN XII reaches the dorsal surface of the hyoglossus muscle, it usually radiates: the lateral branches innervate the anterior part, and the medial branches supply the posterior part of the tongue (Fig. 7) [11].

In this case, the prognosis associated with the remaining dentofacial deformities, such as dental arch misalignment, class III malocclusion, and open bite, is less favorable than is typical. Thus, orthognathic surgery is needed. Since all teeth are still deciduous, planning for growth is essential [7].

Thus, we propose early surgical intervention for macroglossia resulting from irreversible causes like lymphangioma. Since macroglossia growth accompanies that of dento-musculoskeletal structures, enlarged tongue would be the only early structural deformity. In our case, the tongue of the patient was con- tained within his oral cavity, but disproportionate mandibular growth causing drooling and an open bite occurred as significant sequelae. If the patient had been treated earlier, skeletal deformities would not have arisen, and no further treatment would have been necessary.

\section{NOTES}

\section{Conflict of interest}

No potential conflict of interest relevant to this article was reported.

\section{Ethical approval}

The study was approved by the Institutional Review Board of Catholic Medical Center (IRB No. KC19ZESI0217) and performed in accordance with the principles of the Declaration of Helsinki. Written informed consent was obtained.

\section{Patient consent}

The patient's parent provided written informed consent for the publication and the use of his images.

\section{ORCID}

Jun Hyeok Kim https://orcid.org/0000-0003-4657-2090

Hyo Jeong Kwon https://orcid.org/0000-0002-5778-5692

Jong Won Rhie https://orcid.org/0000-0002-9398-8059

\section{REFERENCES}

1. Lam AM, Vavilala MS. Macroglossia: compartment syndrome of the tongue? Anesthesiology 2000;92:1832-5.

2. Topouzelis N, Iliopoulos C, Kolokitha OE. Macroglossia. Int Dent J 2011;61:63-9.

3. Hiiemae KM, Palmer JB. Tongue movements in feeding and speech. Crit Rev Oral Biol Med 2003;14:413-29.

4. Gasparini G, Saltarel A, Carboni A, Maggiulli F, Becelli R. Surgical management of macroglossia: discussion of 7 cases. Oral Surg Oral Med Oral Pathol Oral Radiol Endod 2002;94:56671.

5. Shetty SC, Hasan S, Chary G, Balasubramanya AM, Das UC, Harshad D. Lymphangiomatous macroglossia causing upper airway obstruction and associated Plummer-Vinson syndrome. Otolaryngol Head Neck Surg 2001;124:477-8.

6. Jian XC. Surgical management of lymphangiomatous or lymphangiohemangiomatous macroglossia. J Oral Maxillofac Surg 2005;63:15-9.

7. Wolford LM, Cottrell DA. Diagnosis of macroglossia and indications for reduction glossectomy. Am J Orthod Dentofacial 
Orthop 1996;110:170-7.

8. Friede H, Figueroa AA. The Beckwith-Wiedemann syndrome: a longitudinal study of the macroglossia and dentofacial complex. J Craniofac Genet Dev Biol Suppl 1985;1:179-87.

9. Shangkuan H, Xinghai W, Zengxing W, Shizhen Z, Shiying J, Yishi C. Anatomic bases of tongue flaps. Surg Radiol Anat
1998;20:83-8.

10. Doty RL, Cummins DM, Shibanova A, Sanders I, Mu L. Lingual distribution of the human glossopharyngeal nerve. Acta Otolaryngol 2009;129:52-6.

11. Mu L, Sanders I. Human tongue neuroanatomy: nerve supply and motor endplates. Clin Anat 2010;23:777-91. 